\title{
Use of primary and hospital care health services by chronic patients according to risk level by adjusted morbidity groups
}

Jaime Barrio-Cortes ${ }^{1,2,3^{*}}$, María Soria-Ruiz-Ogarrio ${ }^{4}$, María Martínez-Cuevas ${ }^{5}$, Almudena Castaño-Reguillo ${ }^{4}$, Mariana Bandeira-de Oliveira ${ }^{4}$, María Teresa Beca-Martínez ${ }^{6}$, María Carmen López-Rodríguez ${ }^{4}$ and María Ángeles Jaime-Sisó ${ }^{4}$

\begin{abstract}
Background: Patients with chronic diseases have increased needs for assistance and care. The objective of this study was to describe the characteristics and use of primary care $(\mathrm{PC})$ and hospital care $(\mathrm{HC})$ health services by chronic patients according to risk level based on adjusted morbidity groups (AMG) and to analyze the associated factors.

Methods: Cross-sectional descriptive observational study. Patients from a basic health area classified as chronically ill by the AMG classification system of the Madrid PC electronic medical record were included. Sociodemographic, clinical-care characteristics (classified as predisposing factors or need factors) and service utilization variables were collected. Univariate, bivariate and simple linear regression analyses were performed.

Results: The sample consisted of 9866 chronic patients and 8332 (84.4\%) used health services. Of these service users, 63\% were women, mean age was $55.7(S D=20.8), 439(5.3 \%)$ were high risk, $1746(21.2 \%)$ were medium risk, and 6041 (73.4\%) were low risk. A total of 8226 (98.7\%) were PC users, and $4284(51.4 \%)$ were HC users. The average number of annual contacts with PC was $13.9(S D=15)$; the average number of contacts with $H C$ was $4.8(S D=6.2)$. Predisposing factors associated with services utilization at both care levels were: age (B coefficient $[B C]=0.03$ and $0.018,95 \% \mathrm{Cl}=0.017-0.052$ and $0.008-0.028$, respectively, for $\mathrm{PC}$ and $\mathrm{HC})$ and Spanish origin $(\mathrm{BC}=0.962$ and 3.396, 95\% Cl=0.198-1.726 and 2.722-4.070); need factors included: palliative care $(\mathrm{BC}=10,492$ and 5047; $95 \% \mathrm{Cl}=6457-$ 14,526 and 3098-6995), high risk ( $\mathrm{BC}=4631$ and $2730,95 \% \mathrm{Cl}=3022-6241$ and 1.949-3.512), number of chronic diseases $(B C=1.291$ and $0.222,95 \% \mathrm{Cl}=1.068-1.51$ and $0.103-0.341)$ and neoplasms ( $\mathrm{BC}=2.989$ and $4.309,95 \%$ $\mathrm{Cl}=1.659-4.319$ and $3.629-4.989)$.
\end{abstract}

\footnotetext{
* Correspondence: jaime.barrio@salud.madrid.org

'Primary Care Investigation Unit, Gerencia Asistencial de Atención Primaria, Madrid, Spain

${ }^{2}$ Foundation for Biosanitary Research and Innovation in Primary Care, Madrid, Spain

Full list of author information is available at the end of the article
}

(c) The Author(s). 2021 Open Access This article is licensed under a Creative Commons Attribution 4.0 International License, which permits use, sharing, adaptation, distribution and reproduction in any medium or format, as long as you give appropriate credit to the original author(s) and the source, provide a link to the Creative Commons licence, and indicate if changes were made. The images or other third party material in this article are included in the article's Creative Commons licence, unless indicated otherwise in a credit line to the material. If material is not included in the article's Creative Commons licence and your intended use is not permitted by statutory regulation or exceeds the permitted use, you will need to obtain permission directly from the copyright holder. To view a copy of this licence, visit http://creativecommons.org/licenses/by/4.0/ The Creative Commons Public Domain Dedication waiver (http://creativecommons.org/publicdomain/zero/1.0/) applies to the data made available in this article, unless otherwise stated in a credit line to the data. 
Conclusions: The characteristics and PC and HC service utilization of chronic patients were different and varied according to their AMG risk level. There was greater use of PC services than $\mathrm{HC}$ services, although utilization of both levels of care was high. Service use was related to predisposing factors such as age and country of origin and, above all, to need factors such as immobility, high risk, and number and type of chronic diseases that require follow-up and palliative care.

Keywords: Chronic diseases, Multimorbidity, Risk levels, Morbidity classification, Health services, Primary care, Hospital care

\section{Introduction}

Patients with chronic diseases pose challenges for health systems. These pathologies have multiple impacts, are associated with high morbidity and mortality and are responsible for progressive deterioration, functional limitations, loss of autonomy, reduced quality of life and an increased use of health services, including both primary care $(\mathrm{PC})[1,2]$ and hospital care $(\mathrm{HC})[3,4]$.

In Spain, PC is the first level of care and the gateway to the health system. It is characterized by maximum accessibility and includes professionals in family medicine, pediatrics, nursing, dentistry, and social care work. PC offers comprehensive care that includes health promotion and education in healthy habits, prevention and monitoring of chronic diseases and orientation to social assistance programs. $\mathrm{HC}$ is the second level of care. It offers the most complex and costly diagnostic and/or therapeutic resources within the system (those whose efficiency is very low if they are not concentrated in one location), which are accessed by referral from a PC physician or in cases of urgency or vital risk that may require therapeutic measures that are only available in the hospital environment. $\mathrm{HC}$ fundamentally includes emergency room visits, outpatient visits, inpatient hospitalization, and day $\mathrm{HC}$ [5]. Coordination between the two levels of care has become a priority, especially for chronic patients, as a means of achieving continuity of care, reducing costs and improving the quality of care $[4,6,7]$.

To respond to the needs of these patients, in recent years, multiple strategies to address chronicity have been implemented that seek to improve the care quality and improve the use efficiency of available resources. Many of these strategies are based on models that stratify the chronic population at different risk levels according to the Kaiser Permanente pyramid model (high-risk, medium-risk and low-risk chronic patients) $[8,9]$ or the King's Fund, which includes health promotion and prevention for the general population together with health and social-care integrated vision [10]. For this stratification, morbidity classification systems are used. In Spain, one of the most frequently used classifiers in recent years has been adjusted morbidity groups (AMGs), which were developed with data from our health system and have been integrated into the electronic medical records (EMRs) of several PC autonomous communities [11]. These AMGs constitute a risk adjustment system that classifies individuals by taking their morbidity and complexity into account [8]. This new classification system is comparable to others that are available, such as adjusted clinical groups (ACGs) [12] and clinical risk groups (CRGs) [13], being proved more accurate than the latter for predicting PC visits, hospital admissions and pharmacy spending [14-16].

EMRs include data that allow an analysis of the relationship between the use of services and the factors that influence it, and they are accessible and reliable sources of information [17]. There are several models that provide a theoretical explanation of the health services utilization by system users and the influences of associated factors, but none of them are considered complete or definitive. Andersen [18] developed a behavioral model that explains how health services utilization is determined by a complex interaction of predisposing factors, need factors and facilitating factors. These factors are interrelated and favor or limit the level and frequency of service use [19].

Although there are studies on the usefulness of AMG and the stratification of chronic patients into different risk levels in the general population in PC $[8,20,21]$, there is little information about the differences between $\mathrm{PC}$ and $\mathrm{HC}$ services utilization according to AMG risk levels.

The objective of this study was to describe the characteristics and use of $\mathrm{PC}$ and $\mathrm{HC}$ services of patients with chronic diseases patients according to their AMG risk level and to analyze the need and predisposing factors associated with this use.

\section{Materials and methods}

\section{Study design, setting and sample}

This was a cross-sectional descriptive study with an analytical approach. The study population comprised users from Ciudad Jardín healthcare centre, which has an enrolled population from 18,107 . This centre is located in the Chamartín district in the northern area at the city of Madrid. It had a population of 143,424 with an average age of 45 years (23\% > 65 years); $55 \%$ are women, $8.9 \%$ are foreigners, and the neighborhood has a low degree of socioeconomic deprivation [22, 23]. The study period 
was between June 2015 and June 2016. The study included patients who had a chronic disease according to the AMG stratification tool that has been integrated into the PC services of the community of Madrid. The chronic diseases considered by the classification tool are described in the Community of Madrid Strategy of Care for Patients with Chronic Diseases [24] and are reflected in Additional file 1: Appendix I. The AMG take into consideration several indicators to measure complexity based on morbidity (CBM): mortality, income (urgent, scheduled, medical and surgical), visits in primary care, external consultation, emergencies (primary care - specialized), prescription, outpatient hospital medication, day hospital, stays in social health centers, mental health. Through statistical modelling assign in each patient a numerical value of complexity (relative weight), which is the one that determines the risk level within assigning three cut-off points obtained from percentiles of the entire population $[8,25]$.

\section{Variables}

The following variables for the use of PC services were collected: total annual contacts (a patient was considered a PC user if the number of annual contacts with PC was $\geq 1$ ), type of contact (health, administrative or laboratory), form of contact (face-to-face, telephone, home visit) and professional contacted (family doctor, nurse, pediatrician, social worker, midwife, physiotherapist and dentist). For $\mathrm{HC}$ service use, the following variables were collected: total annual contacts (a patient was considered an $\mathrm{HC}$ user if the number of annual contacts with $\mathrm{HC}$ was $\geq 1$ ), visits to outpatient clinics, emergency room visits, admissions, and day hospital visits.

Predisposing factors included gender, age and country of origin (Spain, Europe and the rest of the world). The need factors that were considered were being immobilized at home, being institutionalized, having a primary caregiver, receiving palliative care [26], number and type of chronic diseases, multimorbidity $(\geq 2$ chronic diseases), risk level (high, medium and low), AMG complexity index [8] and polymedication (patients with a medication regimen that implies having been prescribed five or more drugs for their chronic conditions). The low risk refers to patients with chronic conditions that are still in incipient stages. The medium risk refers to patients with chronic conditions that need disease-based approach. The high risk refers to highly complex patients, with multimorbidity and a multidisciplinary care approach.

This information was collected in PC EMRs and HC EMRs by the health professionals responsible for the patient care. The Madrid Information Health System Information Department extracted and anonymized the information in a database. The sociodemographic and clinical care data available were recorded as of June 30, 2015; for the PC and HC use variables, visits between June 30, 2015, and June 30, 2016, were considered.

\section{Statistical analysis}

Qualitative variables are described as frequencies and percentages or as means with their corresponding standard deviation. The normality of the quantitative variables was determined. For comparisons of qualitative variables, the chi-square test was used, and the MannWhitney and Kruskal-Wallis U tests were used for quantitative variables. The Bonferroni method was used for multiple comparisons. The relationship between the number of annual contacts in $\mathrm{PC}$ and $\mathrm{HC}$; and the predisposing and need factors of the Andersen model was analyzed using linear regression analysis, and an explanatory model was constructed that included factors that were statistically significant $(p<0.05)$ in the bivariate analysis. The final model was selected for its consistency with the theoretical model and according to the principle of parsimony; that is, between two possible similar models, the one that was simpler and required the fewest assumptions for its elaboration was chosen.

\section{Ethics approval}

The study was approved by the Medical Research Ethics Committee of the La Princesa University Hospital and received a favorable report from the Local Research Commission of the Healthcare Directorate Center of Healthcare Management of the Community of Madrid.

\section{Results}

A total of 9866 chronic patients were identified (54.4\% of the total number of people assigned to the center), of whom 1534 (15.5\% of all chronic patients) did not use PC or HC services and 8332 (84.5\%) used these services. The patients who did not use PC or HC services had an average age of 46.7 years, compared to 57.4 years for those who used these services; $52.6 \%$ of nonusers were women, compared to $63 \%$ of users, and $70.9 \%$ of nonusers were of Spanish origin, compared to $83.9 \%$ of users. A total of 0.3 and $0.1 \%$ of patients who were not service users were immobilized and required primary caregivers, respectively, compared to 3.6 and $2.7 \%$ of users. Multimorbidity was present in $35.6 \%$ of non-service users, compared to $65.9 \%$ of users, and $1 \%$ of nonusers were polymedicated, compared to $19 \%$ of users. Among the nonusers, $0.2 \%$ were high-risk patients, compared to $5.3 \%$ of service users, while $97.8 \%$ of nonusers were low risk, compared to $73.3 \%$ of users. The predisposing and need factors of chronic patients according to their use of services are described in Table 1.

Of the total number of service users, 8226 (98.7\%) used PC services, and 4284 (51.4\%) used HC services. 
Table 1 Predisposing and need factors of chronic patients according to their use of services

\begin{tabular}{|c|c|c|c|c|}
\hline $\begin{array}{l}\text { Use of services } \\
\text { n (\%) }\end{array}$ & $\begin{array}{l}\text { Total } \\
9866 \text { (100) }\end{array}$ & $\begin{array}{l}\text { Nonuse } \\
\text { of services } \\
1534 \text { (15.5) }\end{array}$ & $\begin{array}{l}\text { Use of PC and/or } \\
\text { HC services } \\
8332(84.5)\end{array}$ & $p$ \\
\hline \multicolumn{5}{|l|}{ Predisposing factors } \\
\hline Female gender & $6056(61.4)$ & $807(52.6)$ & $5249(63.0)$ & $<0.01$ \\
\hline $\mathrm{Age}^{\mathrm{a}}$ & $55.7(20.8)$ & $46.7(16.4)$ & $57.4(21.1)$ & $<0.01$ \\
\hline$<15$ years & $343(3.5)$ & $38(2.5)$ & $305(3.7)$ & $<0.01$ \\
\hline $15-65$ years & $6040(61.2)$ & $1305(85.1)$ & 4735 (56.8) & \\
\hline$>65$ years & $483(35.3)$ & $191(12.5)$ & $3292(39.5)$ & \\
\hline Origin Spain & 8078 (81.9) & $1087(70.9)$ & $6991(83.9)$ & $<0.01$ \\
\hline Europe & $367(3.7)$ & $107(7.0)$ & $260(3.1)$ & \\
\hline Rest of the world & $1421(14.4)$ & $340(22.2)$ & $1081(13.0)$ & \\
\hline \multicolumn{5}{|l|}{ Need factors } \\
\hline Immobilized & $300(3.0)$ & $4(0.3)$ & $296(3.6)$ & $<0.01$ \\
\hline Institutionalized & $161(1.6)$ & $4(0.3)$ & $157(1.9)$ & $<0.01$ \\
\hline Primary Caregiver & $229(2.3)$ & $1(0.1)$ & $228(2.7)$ & $<0.01$ \\
\hline Home support & $80(0.8)$ & $1(0.1)$ & $79(0.9)$ & $<0.01$ \\
\hline Palliative care & $44(0.4)$ & $2(0.1)$ & $42(0.5)$ & 0.04 \\
\hline Complexity weight $^{a}$ & $6.7(7.0)$ & $2.8(2.6)$ & $7.4(7.4)$ & $<0.01$ \\
\hline High risk & $444(4.5 \%)$ & $3(0.2)$ & $441(5.3)$ & $<0.01$ \\
\hline Medium risk & $1784(18.1 \%)$ & $31(2.0)$ & $1753(21)$ & \\
\hline Low risk & $7638(77.4 \%)$ & $1500(97.8)$ & $6138(73.7)$ & \\
\hline Multimorbidity & $6036(61.2)$ & $546(35.6)$ & $5490(65.9)$ & $<0.01$ \\
\hline Chronic diseases $^{\mathrm{a}}$ & $2.5(1.8)$ & $1.6(.9)$ & $2.7(1.9)$ & $<0.01$ \\
\hline Anemia & $908(9.2)$ & $136(8.9)$ & $772(9.3)$ & 0.6 \\
\hline Anxiety & $2345(23.8)$ & $356(23.2)$ & 1989 (23.9) & 0.6 \\
\hline Osteoarthritis & $1055(10.7)$ & $50(3.3)$ & $1005(12.1)$ & $<0.01$ \\
\hline Asthma & $1044(10.6)$ & $177(11.5)$ & $867(14.4)$ & 0.1 \\
\hline Ischemic heart disease & $370(3.8)$ & $14(0.9)$ & $356(4.3)$ & $<0.01$ \\
\hline Cirrhosis & $479(4.9)$ & $47(3.1)$ & $432(5.2)$ & $<0.01$ \\
\hline Dementia & $213(2.2)$ & $5(0.3)$ & $208(2.5)$ & $<0.01$ \\
\hline Depression & $1251(12.7)$ & $133(8.7)$ & $1118(13.4)$ & $<0.01$ \\
\hline Diabetes mellitus & $1063(10.8)$ & $57(3.7)$ & $1006(12.1)$ & $<0.01$ \\
\hline Dyslipidemia & $3780(38.3)$ & $379(24.7)$ & $3401(40.8)$ & $<0.01$ \\
\hline Dysrhythmias & $696(7.1)$ & $40(2.6)$ & $656(7.9)$ & $<0.01$ \\
\hline OCPD & $389(3.9)$ & $13(0.8)$ & $376(4.5)$ & $<0.01$ \\
\hline Glaucoma & $395(4.0)$ & $18(1.2)$ & $377(4.5)$ & $<0.01$ \\
\hline Hypertension & $3418(34.6)$ & $270(17.6)$ & 3148 (37.8) & $<0.01$ \\
\hline Stroke & $267(2.7)$ & $11(0.7)$ & $256(3.1)$ & $<0.01$ \\
\hline Heart failure & $240(2.4)$ & $5(0.3)$ & $235(2.8)$ & $<0.01$ \\
\hline Chronic kidney disease & $142(1.4)$ & $1(0.1)$ & $141(1.7)$ & $<0.01$ \\
\hline Active neoplasia & $481(4.9)$ & $25(1.6)$ & $456(5.5)$ & $<0.01$ \\
\hline Obesity & $1626(16.5)$ & $209(13.6)$ & $1417(17)$ & $<0.01$ \\
\hline Thyroid disorder & $1646(16.7)$ & $171(11.1)$ & $1474(17.7)$ & $<0.01$ \\
\hline HIV & $55(0.6)$ & $5(0.3)$ & $50(0.6)$ & 0.2 \\
\hline Polymedication & $1598(16.2)$ & $16(1.0)$ & $1582(19.0)$ & $<0.01$ \\
\hline
\end{tabular}

${ }^{\mathrm{a}}$ Mean (standard deviation) 
Among the PC users, $63.2 \%$ were women, the average age was 57.5 years, and $83 \%$ were Spanish; among the $\mathrm{HC}$ users, $61.3 \%$ were women, the average age was 60.4 years, and 93\% were Spanish. A total of 3.6 and 2.8\% were immobilized and had a primary caregiver compared in PC compared to 4.5 and $3.6 \%$ of those in HC. A total of 66.1 and $19.2 \%$ of PC users had multimorbidity and polymedication, respectively, compared to 73.4 and $25 \%$ of $\mathrm{HC}$ users, respectively. A total of $5.3 \%$ of PC users were classified as high risk, compared to $8.8 \%$ of $\mathrm{HC}$ users, while $62 \%$ of PC users were considered low risk, versus $73.4 \%$ of $\mathrm{HC}$ users. The predisposing and need factors of chronic patients according to their PC or $\mathrm{HC}$ service use are described in Table 2. These predisposing and need factors of chronic patients who used PC and $\mathrm{HC}$ services are stratified by sex and risk level in Tables 3 and 4 , respectively.

Among the PC users, the average number of annual contacts was 13.9. The average number of healthcare contacts was 11.9. The average number of face-to-face contacts was 12.7. Family doctors were contacted for an average of 7.2 visits, nurses had an average of 3.8 contacts, pediatricians had an average of 0.3 , physical therapists had an average of 0.3 , and social workers had an average of 0.08 . Of the patients who used $\mathrm{HC}$, the average number of annual contacts was 4.8. The average number of visits to outpatient clinics was 3.7, followed by an average of 0.7 visits to the emergency room, 0.3 visits to the day hospital and 0.2 hospitalizations. The use of $\mathrm{PC}$ and $\mathrm{HC}$ services stratified according to risk level, gender and age groups is shown in Table 5.

After multivariate adjustment according to behavioral model factors, female gender was associated with greater use of $\mathrm{PC}$ services $(\mathrm{B}$ coefficient $(\mathrm{BC})=0.675,95 \% \mathrm{CI}=$ $0.082 ; 1.268)$, age $(B C=0.035,95 \% C I=0.017 ; 0.052)$, as was Spanish origin $(\mathrm{BC}=0.962,95 \% \mathrm{CI}=0.198 ; 1.726)$, immobilization $(\mathrm{BC}=8.129 ; 95 \% \mathrm{CI}=6.437 ; 9.822)$, the need for palliative care $(\mathrm{BC}=10.492 ; 95 \% \mathrm{CI}=6.457$; 14.526); high risk level $(\mathrm{BC}=4.631 ; 95 \% \mathrm{CI}=3.022$; 6.241), number of chronic diseases $(\mathrm{BC}=1.291 ; 95 \% \mathrm{CI}=$ $1.068 ; 1.510)$, diabetes mellitus $(\mathrm{BC}=2.332,95 \% \mathrm{CI}=$ $1.375 ; 3.290)$, heart failure $(\mathrm{BC}=3243 ; 95 \% \mathrm{CI}=1361$; $5125)$, stroke $(B C=3415,95 \% C I=1707 ; 5122)$, dementia $(B C=5267 ; 95 \% C I=1376 ; .157)$, neoplasia $(B C=$ 2,89); $95 \% \mathrm{CI}=1.659 ; 4.319)$ and polymedication $(\mathrm{BC}=$ 6.600; $95 \% \mathrm{CI}=5.649 ; 7.552)$. Age $(\mathrm{BC}=0.018,95 \% \mathrm{CI}=$ 0.017; 0.052), Spanish origin $(B C=3.396,95 \% C I=2.722$; $4.070)$, and immobilization $(\mathrm{BC}=-1.522 ; 95 \% \mathrm{CI}=-$ 2.422 ; -0 ) were associated with the $\mathrm{HC}$ model, 62 ), as were the need for palliative care $(\mathrm{BC}=5.047 ; 95 \% \mathrm{CI}=$ $3.098 ; 6.995)$, high risk level $(\mathrm{BC}=2.730 ; 95 \% \mathrm{CI}=1.949$; 3.512), number of chronic diseases $(\mathrm{BC}=0.222 ; 95 \% \mathrm{CI}=$ 0.103; 0.341), $\mathrm{COPD}(\mathrm{BC}=1.34995 \% \mathrm{CI}=0.616 ; 2.082)$, depression $(\mathrm{BC}=0.723 ; 95 \% \mathrm{CI}=0.221 ; 1.220)$, dementia
$(\mathrm{BC}=-1.504 ; 95 \% \mathrm{CI}=-2.560 ;-0.448)$ and active neoplasia $(\mathrm{BC}=4.309 ; 95 \% \mathrm{CI}=3.629 ; 4.989)$ (Table 6).

\section{Discussion}

A large majority of the chronic patients were health service users. These service users were older, with a predominance of female gender and Spanish nationality, greater morbidity, increased presence of serious diseases and a greater need for assistance, care and medication than those who did not use services. The use of both PC and $\mathrm{HC}$ services was high, with greater use of PC than $\mathrm{HC}$, and an increase in the use of both levels of care with increasing risk level and age. The factors associated with the use of both PC and $\mathrm{HC}$ included predisposing factors, such as age and Spanish origin, and need-related factors, such as the need for palliative care, high risk, number of chronic diseases and neoplasms.

\section{Characteristics of the patient population and the use of $\mathrm{PC}$ and $\mathrm{HC}$ services}

The chronic patients had a high average age and a predominance of Spanish nationality. Almost two-thirds were women and had multimorbidity (the most frequent of which chronic cardiovascular diseases, osteoarticular diseases and neoplasms), and more than a quarter were polymedicated. These data can be considered representative of the Community of Madrid, and their distribution is correlated with the total stratification pyramid published by the Community [11] and is similar to others European studies with similar objectives [27-29].

A large majority of chronic patients used PC services, and patients at all risk levels had a high average number of annual contacts with PC. The majority of the contacts were health contacts and took place in person, as observed in other studies [30-32]. The low number of non-face-to-face contacts and home visits stood out; this is a potential area of care for chronic patients that should be strengthened, particularly in light of the COVID-19 pandemic. The average number of contacts with family doctors in our study was lower than the average number of physician consultations reported in comparable studies [33, 34]. However, it was almost double number of contacts with nurses, unlike previous studies in which the average number of contacts with nurses was higher than with doctors [35]. This lower number of nursing contacts in our study is striking and should prompt a reflection on the care provided for these patients, since strategies for addressing chronicity which postulate models of care that enhance and prioritize care and follow-up by the PC nursing staff and reserve contact with the $\mathrm{PC}$ family physician for issues that require medical attention [24]. Along this line, despite the good socioeconomic indicators of the health service area in general, it would be expected that some 
Table 2 Predisposing and need factors of chronic patients according to their use of Primary Care (PC) or Hospital care (HC) services

\begin{tabular}{|c|c|c|c|c|}
\hline $\begin{array}{l}\text { Use of services } \\
\text { n (\%) }\end{array}$ & $\begin{array}{l}\text { Use of PC services } \\
8226(98.7)\end{array}$ & $95 \% \mathrm{Cl}$ & $\begin{array}{l}\text { Use of HC services } \\
4284(51.4)\end{array}$ & $95 \% \mathrm{Cl}$ \\
\hline \multicolumn{5}{|l|}{ Predisposing factors } \\
\hline Female gender & $5201(63.2)$ & $62.2-64.3$ & $2626(61.3)$ & $59.8-62.7$ \\
\hline $\mathrm{Age}^{\mathrm{a}}$ & $57.5(21.1)$ & $57.0-58.0$ & $60.4(20.4)$ & $59.8-70.0$ \\
\hline$<15$ years & $303(3.7)$ & $3.3-4.1$ & $114(2.7)$ & $2.1-3.1$ \\
\hline $15-65$ years & $4643(56.4)$ & $55.4-57.5$ & $2236(52.2)$ & $50.7-53.7$ \\
\hline$>65$ years & 3280 (39.9) & $38.2-40.9$ & $1934(45)$ & $43.6-46.6$ \\
\hline Origin Spain & $6898(83.9)$ & $83.1-84.6$ & 3,85 (93) & $92.2-93.4$ \\
\hline Europe & $256(3.1)$ & $2.7-3.5$ & $81(1.9)$ & $1.5-2.3$ \\
\hline Rest of the world & $1072(13.0)$ & $12.3-13.8$ & $218(5.1)$ & $4.4-5.7$ \\
\hline \multicolumn{5}{|l|}{ Need factors } \\
\hline Immobilized & 295 (3.6) & $3.2-4.0$ & $194(4.5)$ & $3.9-5.1$ \\
\hline Institutionalized & $157(1.9)$ & $1.6-2.2$ & $88(2.1)$ & $1.6-2.5$ \\
\hline Primary caregiver & $227(2.8)$ & $2.4-3.1$ & $154(3.6)$ & $3.0-4.1$ \\
\hline Home support & $78(0.9)$ & $0.7-1.1$ & $50(1.2)$ & $0.8-1.5$ \\
\hline Palliative care & $42(0.5)$ & $0.3-0.7$ & $35(0.8)$ & $0.5-1.1$ \\
\hline Complexity weight $^{a}$ & $7.5(7.3)$ & $7.3-7.6$ & $9.4(8.6)$ & $9.1-9.6$ \\
\hline High risk & $439(5.3)$ & $4.8-5.8$ & $376(8.8)$ & $7.9-9.6$ \\
\hline Medium risk & $1746(21.2)$ & $20.3-22.1$ & $1251(29.2)$ & $27.8-30.6$ \\
\hline Low risk & $6041(73.4)$ & $72.2-74.4$ & $2657(62)$ & $60.6-63.5$ \\
\hline Multimorbidity & $5541(66.1)$ & $65.1-67.2$ & $3145(73.4)$ & $72.1-74.7$ \\
\hline Chronic diseases $^{\mathrm{a}}$ & $2.7(1.88)$ & $2.6-2.8$ & $3.1(2.1)$ & $3.0-3.2$ \\
\hline Anemia & $763(9.3)$ & $8.6-9.9$ & $401(9.4)$ & $8.5-10.2$ \\
\hline Anxiety & $1962(23.9)$ & $22.9-24.8$ & $1025(23.9)$ & $22.6-25.2$ \\
\hline Osteoarthritis & $996(12.1)$ & $11.4-12.8$ & $638(14.9)$ & $13.8-15.9$ \\
\hline Asthma & $853(10.4)$ & $9.7-11.0$ & $456(10.6)$ & $9.7-11.6$ \\
\hline Ischemic heart disease & $354(4.3)$ & $3.8-4.7$ & $260(6.1)$ & $5.3-6.8$ \\
\hline Cirrhosis & $432(5.3)$ & $4.8-5.7$ & $271(6.3)$ & $5.5-7$ \\
\hline Dementia & $206(2.5)$ & $2.2-2.8$ & $127(3)$ & $2.4-3.5$ \\
\hline Depression & 1108 (13.5) & $12.7-14.2$ & $628(14.7)$ & $13.6-15.7$ \\
\hline Diabetes mellitus & $1004(12.2)$ & $11.5-12.9$ & $630(14.7)$ & $13.6-15.8$ \\
\hline Dyslipidemia & $3377(41.1)$ & $40.0-42.1$ & $1917(44.7)$ & $43.2-46.2$ \\
\hline Dysrhythmias & $651(7.9)$ & $7.3-8.5$ & $449(10.5)$ & $9.6-11.4$ \\
\hline COPD & $374(4.5)$ & $4.1-5.0$ & $275(6.4)$ & $5.7-7.1$ \\
\hline Glaucoma & $374(4.5)$ & $4.1-5.0$ & $247(5.8)$ & $5.1-6.5$ \\
\hline Hypertension & $3129(38)$ & $3.0-39.1$ & $1842(43)$ & $41.5-44.4$ \\
\hline Stroke & $255(3.1)$ & $2.7-3.5$ & $177(4.1)$ & $3.5-4.7$ \\
\hline Heart failure & $234(2.8)$ & $2.5-3.2$ & $183(4.3)$ & $3.7-4.9$ \\
\hline Chronic kidney disease & $141(1.7)$ & $1.4-2.0$ & $116(2.7)$ & $2.2-3.2$ \\
\hline Active neoplasia & $451(5.5)$ & $5.0-6.0$ & $341(8)$ & $7.1-8.8$ \\
\hline Obesity & $1408(17.1)$ & $16.3-18.0$ & $813(19)$ & $17.8-20.0$ \\
\hline Thyroid disorder & $1464(17.8)$ & $17.0-18.6$ & $812(19)$ & $17.8-20.0$ \\
\hline HIV & $46(0.6)$ & $0.5-0.7$ & $40(0.9)$ & $0.6-1.2$ \\
\hline Polymedication & $1581(19.2)$ & $18.4-20.1$ & $1073(25)$ & $23.7-26.3$ \\
\hline
\end{tabular}


Table 3 Predisposing and need factors of chronic patients using primary care (PC) by adjusted morbidity groups (AMG) risk levels and sex

\begin{tabular}{|c|c|c|c|c|c|c|c|}
\hline $\begin{array}{l}\text { PC users } \\
\text { n (\%) }\end{array}$ & $\begin{array}{l}\text { High risk } \\
439(5.3)\end{array}$ & $\begin{array}{l}\text { Medium risk } \\
1746(21.2)\end{array}$ & $\begin{array}{l}\text { Low risk } \\
6041(73.4)\end{array}$ & $P$ & $\begin{array}{l}\text { Female sex } \\
5201(63.2)\end{array}$ & $\begin{array}{l}\text { Male sex } \\
3025(36.8)\end{array}$ & $p$ \\
\hline \multicolumn{8}{|l|}{ Predisposing factors } \\
\hline Female gender & $232(52.8)$ & $1131(64.8)$ & $3838(63.5)$ & $<0.01$ & 100 & 0 & - \\
\hline $\mathrm{Age}^{\mathrm{a}}$ & $77.8(12.8)$ & $72.3(14.9)$ & $51.7(20.1)$ & $<0.01$ & $58.56(20.9)$ & $55.7(21.3)$ & $<0.01$ \\
\hline$<15$ years & $1(0.2)$ & $13(0.7)$ & $289(4.8)$ & & $135(2.6)$ & $168(5.6)$ & $<0.01$ \\
\hline $18-65$ years & $68(15.5)$ & $443(25.4)$ & $4132(68.4)$ & $<0.01$ & 2906 (55.9) & $1737(57.4)$ & \\
\hline$>65$ years & $370(84.3)$ & $1290(73.9)$ & $1620(26.8)$ & & $2160(41.5)$ & $1120(37)$ & \\
\hline Origin Spain & $383(87.2)$ & $1542(88.3)$ & $4973(82.3)$ & & 4363 (83.9) & 2535 (83.8) & 0.1 \\
\hline Europe & $8(1.8)$ & $45(2.6)$ & $203(3.4)$ & $<0.01$ & $176(3.4)$ & $80(2.6)$ & \\
\hline Rest of the world & 48 (10.9) & $169(9.1)$ & $865(14.3)$ & & $662(12.7)$ & $410(13.6)$ & \\
\hline \multicolumn{8}{|l|}{ Need factors } \\
\hline Immobilized & $123(28)$ & $125(7.2)$ & $47(0.8)$ & $<0.01$ & $222(4.3)$ & $73(2.4)$ & $<0.01$ \\
\hline Institutionalized & $42(9.6)$ & $51(2.9)$ & $64(1.1)$ & $<0.01$ & $119(2.3)$ & $38(1.3)$ & $<0.01$ \\
\hline Primary caregiver & $100(22.8)$ & $101(5.8)$ & $26(0.4)$ & $<0.01$ & $164(3.2)$ & $63(2.1)$ & $<0.01$ \\
\hline Home support & $27(6.2)$ & $38(2.2)$ & $13(0.2)$ & $<0.01$ & $58(1.1)$ & $20(0.7)$ & 0.04 \\
\hline Palliative care & $28(6.4)$ & $6(0.3)$ & $8(0.1)$ & $<0.01$ & $19(0.4)$ & $23(0.8)$ & 0.01 \\
\hline Complexity weight $^{a}$ & $30.4(12.5)$ & $12.47(2.7)$ & $4.36(2.2)$ & $<0.01$ & 7. $3(6.7)$ & $7.7(8.3)$ & 0.02 \\
\hline Multimorbidity & $436(99.3)$ & $1,687(96.6)$ & $3318(54.9)$ & $<0.01$ & $3508(67.4)$ & $1933(63.9)$ & $<0.01$ \\
\hline Chronic diseases $^{\mathrm{a}}$ & $6.7(2.4)$ & $4.3(1.5)$ & $1.9(1.1)$ & $<0.01$ & $2.8(1.9)$ & $2.6(1.8)$ & $<0.01$ \\
\hline Anemia & $116(26.4)$ & $163(9.3)$ & $484(8)$ & $<0.01$ & $593(11.4)$ & $170(5.6)$ & $<0.01$ \\
\hline Anxiety & $84(19.1)$ & $417(23.9)$ & $1461(24.2)$ & $<0.01$ & $1438(27.6)$ & $524(17.3)$ & $<0.01$ \\
\hline Osteoarthritis & $108(24.6)$ & $424(24.3)$ & $464(7.7)$ & $<0.01$ & $771(14.8)$ & $225(7.4)$ & $<0.01$ \\
\hline Asthma & $26(5.9)$ & $166(9.5)$ & $661(10.9)$ & 0,06 & $550(10.6)$ & $303(10)$ & 0.4 \\
\hline Ischemic heart disease & $109(24.8)$ & $168(9.6)$ & $77(1.3)$ & $<0.01$ & $131(2.5)$ & $223(7.4)$ & $<0.01$ \\
\hline Cirrhosis & $50(11.4)$ & $184(10.5)$ & $198(3.3)$ & $<0.01$ & $227(4.4)$ & $205(6.8)$ & $<0.01$ \\
\hline Dementia & $55(12.5)$ & $90(5.2)$ & $61(1)$ & $<0.01$ & $160(3.1)$ & $46(1.5)$ & $<0.01$ \\
\hline Depression & $101(23)$ & $382(21.9)$ & $625(10.3)$ & $<0.01$ & $862(16.6)$ & $246(8.1)$ & $<0.01$ \\
\hline Diabetes mellitus & $187(42.6)$ & $425(24.3)$ & $2016(33.4)$ & $<0.01$ & $515(9.9)$ & $489(16.2)$ & $<0.01$ \\
\hline Dyslipidemia & $296(67.4)$ & $1065(61)$ & $2016(33.4)$ & $<0.01$ & $2028(39)$ & $1349(44.6)$ & $<0.01$ \\
\hline Dysrhythmias & $189(43.1)$ & $296(17)$ & $166(2.7)$ & $<0.01$ & $348(7.4)$ & $267(8.8)$ & 0.02 \\
\hline COPD & $108(24.6)$ & $164(9.4)$ & $102(1.7)$ & $<0.01$ & $165(3.2)$ & $209(6.9)$ & $<0.01$ \\
\hline Glaucoma & $44(10)$ & $155(8.9)$ & $175(2.9)$ & $<0.01$ & $125(4.1)$ & $249(4.8)$ & 0.2 \\
\hline Hypertension & $362(82.5)$ & $1177(67.4)$ & $1590(26.3)$ & $<0.01$ & $1872(36)$ & $1257(41.6)$ & $<0.01$ \\
\hline Stroke & $91(20.7)$ & $110(6.3)$ & $54(0.9)$ & $<0.01$ & $141(2.7)$ & $114(3.8)$ & $<0.01$ \\
\hline Heart failure & $122(27.8)$ & $99(5.7)$ & $13(0.2)$ & $<0.01$ & $147(2.8)$ & $87(2.9)$ & 0.9 \\
\hline Chronic kidney disease & $96(21.9)$ & $36(2.1)$ & $9(0.1)$ & $<0.01$ & $70(1.3)$ & $71(2.3)$ & $<0.01$ \\
\hline Active neoplasia & 165 (37.6) & $178(10.2)$ & $108(1.8)$ & $<0.01$ & $288(4.4)$ & $223(7.4)$ & $<0.01$ \\
\hline Obesity & $131(29.8)$ & $455(26.1)$ & 822 (13.6) & $<0.01$ & 861 (16.6) & $547(18.1)$ & 0.08 \\
\hline Thyroid disorder & $107(24.4)$ & $399(22.9)$ & 958 (15.9) & $<0.01$ & 1227 (23.6) & $237(7.8)$ & $<0.01$ \\
\hline HIV & $5(1.1)$ & $11(0.6)$ & $30(0.5)$ & 0.3 & $7(0.1)$ & $39(1.3)$ & $<0.01$ \\
\hline Polymedication & 348 (79.3) & 767 (43.9) & $466(7.7)$ & $<0.01$ & $1090(21)$ & $491(16.2)$ & $<0.01$ \\
\hline
\end{tabular}


Table 4 Predisposing and need factors of chronic patients using hospital care by adjusted morbidity groups (AMG) risk levels and

\begin{tabular}{|c|c|c|c|c|c|c|c|}
\hline $\begin{array}{l}\text { Hospital care users } \\
\text { n (\%) }\end{array}$ & $\begin{array}{l}\text { High risk } \\
376(8.8)\end{array}$ & $\begin{array}{l}\text { Medium risk } \\
1251 \text { (29.2) }\end{array}$ & $\begin{array}{l}\text { Low risk } \\
2657(62)\end{array}$ & $P$ & $\begin{array}{l}\text { Female sex } \\
2626(61.3)\end{array}$ & $\begin{array}{l}\text { Male sex } \\
1658(38.7)\end{array}$ & $p$ \\
\hline \multicolumn{8}{|l|}{ Predisposing factors } \\
\hline Female gender & $198(52.7)$ & $794(63.5)$ & $1634(61.5)$ & & $2626(61.3)$ & 0 & \\
\hline $\mathrm{Age}^{\mathrm{a}}$ & $78(12.2)$ & $71.9(14.7)$ & $52.4(19.5)$ & $<0.01$ & $61.2(20.4)$ & $59,1(20.4)$ & $<0.01$ \\
\hline$<15$ years & $1(0.3)$ & $9(0.7)$ & $104(3.9)$ & & $52(2)$ & $62(3.7)$ & $<0.01$ \\
\hline $15-65$ years & $57(15.2)$ & $329(26.3)$ & $1850(69.6)$ & $<0.01$ & $1344(51.2)$ & $892(53.8)$ & \\
\hline$>65$ years & 318 (84.6) & 913 (73) & $703(26.5)$ & & $1230(46.8)$ & $704(42.5)$ & \\
\hline Origin Spain & $341(90.1)$ & $1181(94.4)$ & 2463(92.7) & 0.032 & $2460(93.7)$ & $1525(92)$ & 0.01 \\
\hline Europe & $6(1.6)$ & $23(1.8)$ & $52(2)$ & & $53(2)$ & $28(1.7)$ & \\
\hline Rest of the world & $29(7.7)$ & $47(3.8)$ & $142(5.3)$ & & $113(4.3)$ & $105(6.3)$ & \\
\hline \multicolumn{8}{|l|}{ Necessity factors } \\
\hline Immobilized & $96(25.5)$ & $75(6)$ & $23(0.9)$ & $<0.01$ & $138(5.3)$ & $56(3.4)$ & $<0.01$ \\
\hline Institutionalized & $31(8.2)$ & $27(2.2)$ & $30(1.1)$ & $<0.01$ & $65(2.5)$ & $23(1.4)$ & 0.014 \\
\hline Primary caregiver & $80(21.3)$ & $61(4.9)$ & $13(0.5)$ & $<0.01$ & $106(4)$ & $48(2.9)$ & 0.051 \\
\hline Home support & $22(5.9)$ & $24(1.9)$ & $4(0.2)$ & $<0.01$ & $31(1.2)$ & $19(1.1)$ & 0.92 \\
\hline Palliative care & $25(6.6)$ & $5(0.4)$ & $5(0.2)$ & $<0.01$ & $16(0.6)$ & $19(1.1)$ & 0.057 \\
\hline Complexity weight $^{a}$ & $30.6(12.6)$ & $12.7(2.7)$ & $4.8(2.2)$ & $<0.01$ & $9.2(7.9)$ & $9.7(9.5)$ & 0.41 \\
\hline Multimorbidity & $372(98.9)$ & $1207(96.5)$ & $1566(58.9)$ & $<0.01$ & $1962(74.7)$ & $1183(71.4)$ & 0.015 \\
\hline Chronic diseases $^{a}$ & $6.9(2.4)$ & $4.3(1.5)$ & $2.1(1.2)$ & $<0.01$ & $3.2(2.1)$ & $3.01(2.02)$ & $<0.01$ \\
\hline Anemia & $99(26.3)$ & $115(9.2)$ & $187(7)$ & $<0.01$ & $292(11.1)$ & $109(6.6)$ & $<0.01$ \\
\hline Anxiety & $75(19.9)$ & $289(23.1)$ & $661(24.9)$ & 0.08 & $732(27.9)$ & $293(17.7)$ & $<0.01$ \\
\hline Osteoarthritis & $96(25.5)$ & $325(26)$ & $217(8.2)$ & $<0.01$ & $476(18.1)$ & $162(9.8)$ & $<0.01$ \\
\hline Asthma & $25(6.6)$ & $120(9.6)$ & $311(11.7)$ & $<0.01$ & $315(12)$ & $141(8.5)$ & $<0.01$ \\
\hline Ischemic heart disease & $92(24.5)$ & $124(9.9)$ & $44(1.7)$ & $<0.01$ & $93(3.5)$ & $167(10.1)$ & $<0.01$ \\
\hline Cirrhosis & $44(11.7)$ & $133(10.6)$ & $94(3.5)$ & $<0.01$ & $139(5.3)$ & $132(8)$ & $<0.01$ \\
\hline Dementia & $47(12.5)$ & $54(4.3)$ & $26(1)$ & $<0.01$ & $91(3.5)$ & $36(2.2)$ & 0.015 \\
\hline Depression & $88(23.4)$ & $252(20.1)$ & $288(10.8)$ & $<0.01$ & $478(18.2)$ & $150(9)$ & $<0.01$ \\
\hline Diabetes mellitus & $156(41.5)$ & $296(23.7)$ & $178(6.7)$ & $<0.01$ & $308(11.7)$ & $322(19.4)$ & $<0.01$ \\
\hline Dyslipidemia & $255(67.8)$ & $758(60.6)$ & $904(34)$ & $<0.01$ & $1134(43.2)$ & $783(47.2)$ & 0.01 \\
\hline Dysrhythmias & $166(44.1)$ & $211(16.9)$ & $72(2.7)$ & $<0.01$ & $257(9.8)$ & $192(11.6)$ & 0.062 \\
\hline COPD & $95(25.3)$ & $124(9.9)$ & $56(2.1)$ & $<0.01$ & $114(4.3)$ & $161(9.7)$ & $<0.01$ \\
\hline Glaucoma & $39(10.4)$ & $106(8.5)$ & $102(3.8)$ & $<0.01$ & $161(6.1)$ & $86(5.2)$ & 0.197 \\
\hline Hypertension & $316(84)$ & $828(66.2)$ & $698(26.3)$ & $<0.01$ & $1084(41.3)$ & $758(45.7)$ & $<0.01$ \\
\hline Stroke & $80(21.3)$ & $69(5.5)$ & $28(1.1)$ & $<0.01$ & $98(3.7)$ & $79(4.8)$ & 0.098 \\
\hline Heart failure & $110(29.3)$ & $67(5.4)$ & $6(0.2)$ & $<0.01$ & $117(4.5)$ & $66(4)$ & 0.45 \\
\hline Chronic kidney disease & $84(22.3)$ & $26(2.1)$ & $6(0.2)$ & $<0.01$ & $58(2.2)$ & $58(3.5)$ & 0.011 \\
\hline Active neoplasia & $144(38.3)$ & $137(11)$ & $60(2.3)$ & $<0.01$ & $156(5.9)$ & $185(11.2)$ & $<0.01$ \\
\hline Obesity & $112(29.8)$ & $322(25.7)$ & $379(14.3)$ & $<0.01$ & 495 (18.8) & 318 (19.2) & 0.789 \\
\hline Thyroid disorder & $91(24.2)$ & $279(22.3)$ & $442(16.6)$ & $<0.01$ & $671(25.6)$ & $141(8.5)$ & $<0.01$ \\
\hline HIV & $5(1.3)$ & $7(0.6)$ & $28(1.1)$ & 0.22 & $4(0.2)$ & $36(2.2)$ & $<0.01$ \\
\hline Polymedication & 307 (81.6) & $541(43.2)$ & $225(8.5)$ & $<0.01$ & 708 (27) & $365(22)$ & $<0.01$ \\
\hline
\end{tabular}




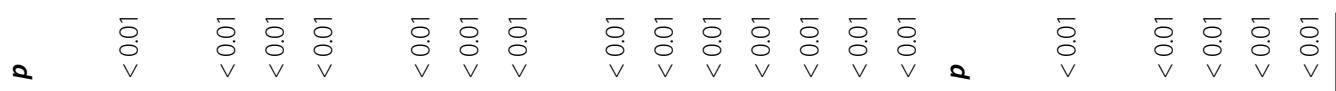

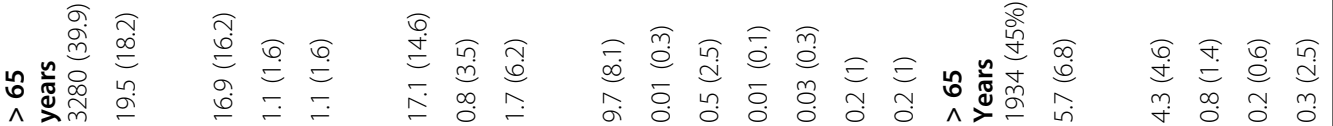
$\frac{9}{3}$

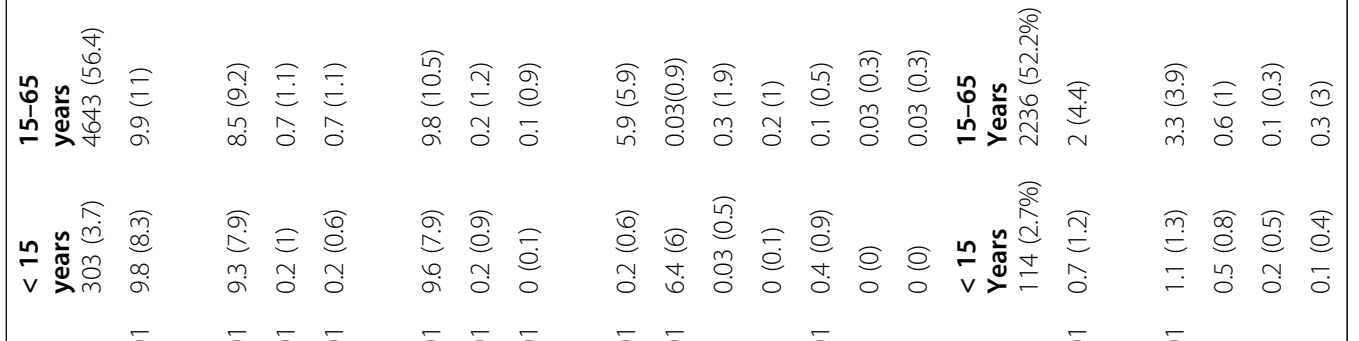

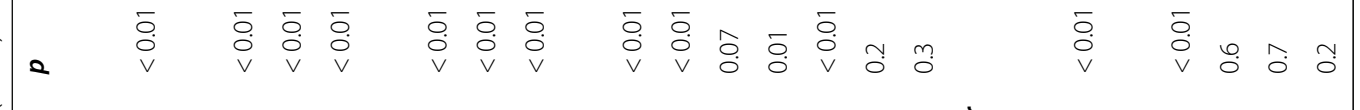

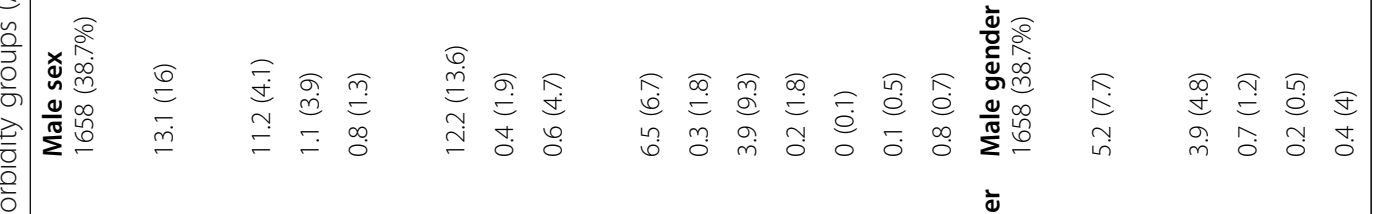

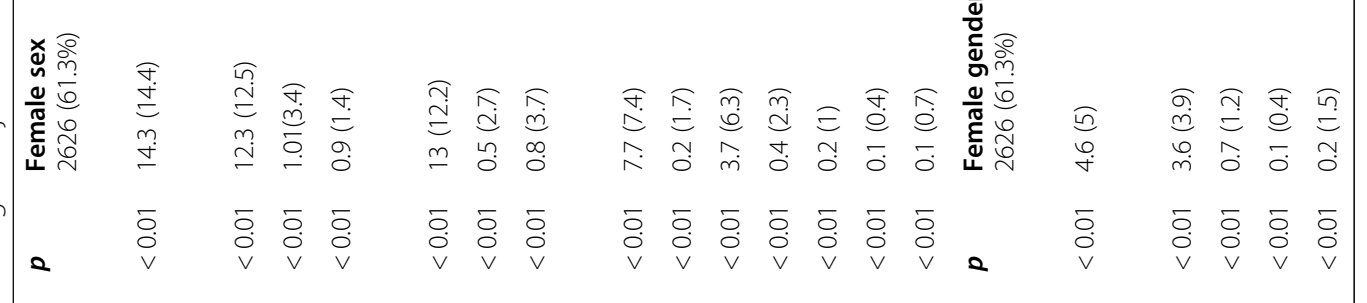

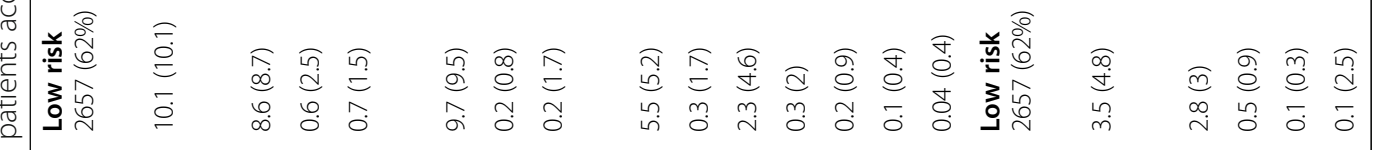

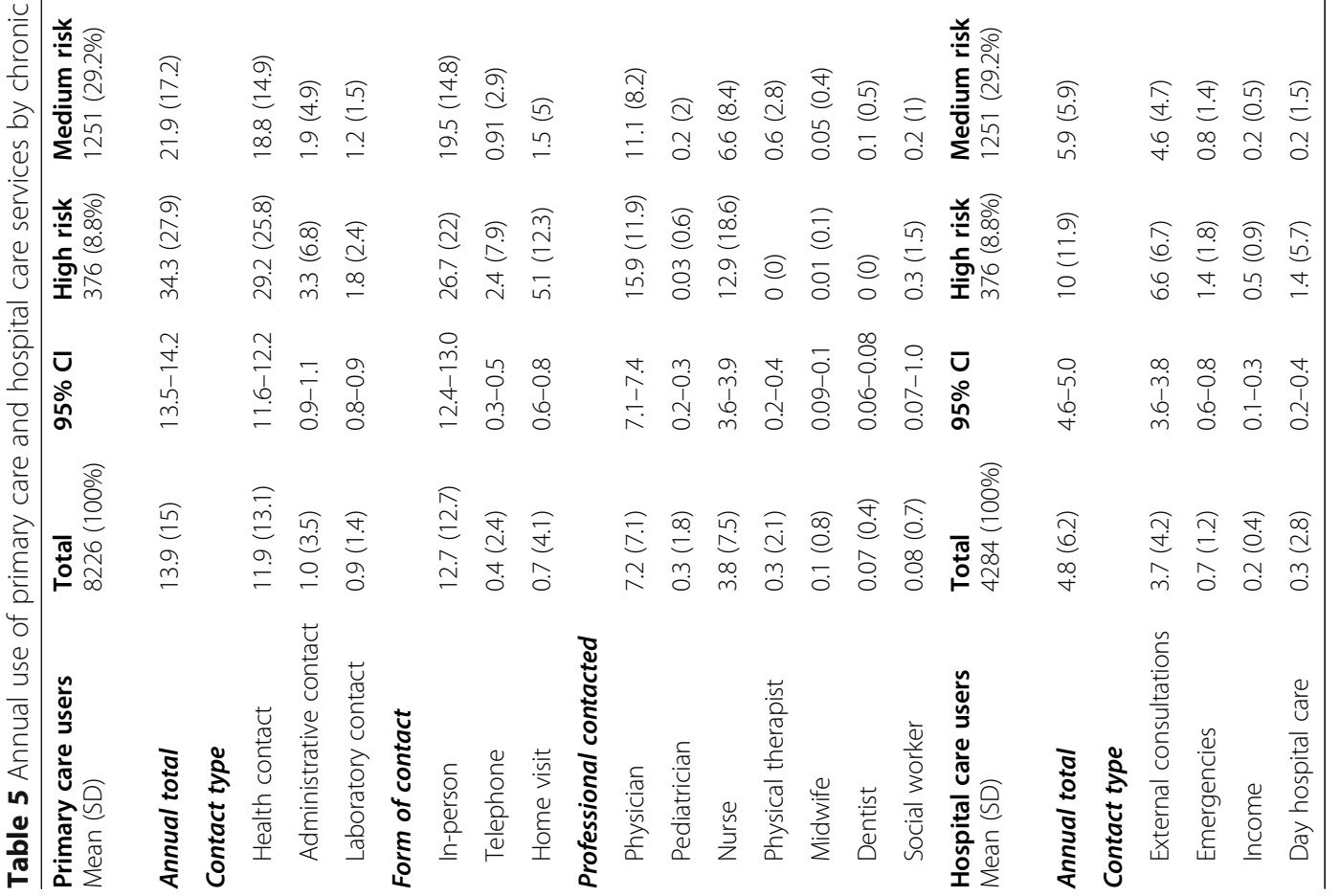


Table 6 Factors associated with chronic patients' use of primary care and hospital care

\begin{tabular}{|c|c|c|}
\hline Predisposing and need factors & B coefficient $(95 \% \mathrm{Cl})$ & $p$ \\
\hline \multicolumn{3}{|l|}{ Primary care users } \\
\hline Female gender & $0.675(0.082 ; 1.268)$ & 0.000 \\
\hline $\mathrm{Age}^{\mathrm{a}}$ & $0.035(0.017 ; 0.052)$ & 0.026 \\
\hline Spanish origin & $0.962(0.198 ; 1.726)$ & 0.014 \\
\hline Immobilized & $8.129(6.437 ; 9.822)$ & 0.014 \\
\hline Palliative care & $10.492(6.457 ; 14.526)$ & 0.000 \\
\hline High risk level & $4.631(3.022 ; 6.241)$ & 0.000 \\
\hline Number of chronic diseases ${ }^{a}$ & $1.291(1.068 ; 1.51)$ & 0.000 \\
\hline Diabetes mellitus & $2.332(1.375 ; 3.290)$ & 0.000 \\
\hline Heart failure & $3.243(1.361 ; 5.125)$ & 0.000 \\
\hline Stroke & $3.415(1.707 ; 5.122)$ & 0.000 \\
\hline Dementia & $5.267(3.376 ; 7.157)$ & 0.000 \\
\hline Active neoplasia & $2.989(1.659 ; 4.319)$ & 0.000 \\
\hline Polymedication & $6.600(5.649 ; 7.552)$ & 0.000 \\
\hline \multicolumn{3}{|l|}{$R 2=0.254$} \\
\hline \multicolumn{3}{|l|}{ Hospital care users } \\
\hline $\mathrm{Age}^{\mathrm{a}}$ & $0.018(0.008 ; 0.028)$ & 0.001 \\
\hline Spanish origin & $3.396(2.722 ; 4.070)$ & 0.000 \\
\hline Immobilized & $-1.522(-2.422 ;-0.62)$ & 0.001 \\
\hline Palliative care & $5.047(3.098 ; 6.995)$ & 0.000 \\
\hline High risk level & $2.730(1.949 ; 3.512)$ & 0.000 \\
\hline Number of chronic diseases ${ }^{a}$ & $0.222(0.103 ; 0.341)$ & 0.000 \\
\hline Active neoplasia & $4.309(3.629 ; 4.989)$ & 0.000 \\
\hline COPD & $1.349(0.616 ; 2.082)$ & 0.000 \\
\hline Depression & $0.723(0.221 ; 1.22)$ & 0.005 \\
\hline Dementia & $-1.504(-2.560 ;-0.448)$ & 0.005 \\
\hline $\mathrm{R} 2=0.151$ & & \\
\hline
\end{tabular}

${ }^{\mathrm{a}}$ Quantitative variables

chronic patients would have increased needs for social assistance; nonetheless, a very low number of contacts with the social worker was observed. The coverage of several healthcare centers by a single social worker could lead to low accessibility and raises the need to strengthen this ratio to offer comprehensive social health care. Among the PC users, all types and forms of contact and all professional contact increased significantly according to the level of risk; the exception was for physical therapists, midwives and dentists, which could be explained because these professionals usually attend to patients who are younger and have lower risk and lower morbidity. In other studies of patients with similar characteristics to those of our high-risk population, such as complex chronic or multiple pathologies, a high degree of contact with PC professionals is also observed in both developed and developing countries [32, 36-38]. The average number of annual contacts with $\mathrm{PC}$ was higher in women than in men, regardless of their risk level, and women had significantly more health contacts, home visits and contacts with doctors than men did. These results are similar to those of other studies [36, 37]. Among the possible explanations for this increased utilization are that women report a worse perceived state of health and have a higher prevalence of minor affective disorders that can generate the need for health consultations; additionally, women are also often caregivers or heads of the household, which could favor a need to consult health services on behalf of other family members [34]. In contrast, nurse consultations were more frequent among high-risk men, which could be due to less self-care capacity among men, especially those in older age ranges [34]. The results show that increased age was associated with a major number of contacts for most types and forms and with professionals other than pediatricians, midwives, physical therapists and dentists. This is because age is one of the most influential modifiers of utilization, as observed in many series [38-40].

The average use of $\mathrm{HC}$ services by these chronic patients was also very high. The majority of contacts were outpatient consultations, followed by emergencies, day hospital care and hospitalizations. The total number of contacts with $\mathrm{HC}$ for all types of visits increased significantly according to risk level. There is no literature that compares the use of hospital services by patients stratified according to AMG, but these data correlate with what is described in the literature for patients with frailty, functional deterioration or high-complexity needs $[41,42]$, who are similar to high-risk patients; in patients with multimorbidity or multiple pathologies [43, 44], who are similar to medium-risk patients; and in patients with a single chronic disease [1], who are similar to lowrisk patients. Regarding sex, the average number of annual contacts with $\mathrm{HC}$, visits to outpatient clinics and admissions was higher in men, which is in line with the results of studies that show that women are hospitalized significantly less often than men [45]. Regarding age and the use of $\mathrm{HC}$ services, chronic patients under 15 years of age had a lower average use of services, while use increased among patients aged 15 to 65 years and was the highest among those older than 65 years, as observed in other studies [42, 44, 46]. This high use of PC and general $\mathrm{HC}$ services can be favored by inadequate management of resources and a lack of care continuity, as has been identified by studies in our environment $[6,47]$. To improve continuity of care, transformative and innovative leadership by organizations is necessary, as seen in evidence from the United States, Europe and Spain from new care models for chronic patients that have increased the quality, efficiency and sustainability of health systems, improved health outcomes and reduced the 
number of emergency room visits, admissions and readmissions [48].

\section{Factors associated with the utilization of services}

According to the literature, the use of health services depends mainly on the user's predisposing and need factors [49]. Based on the Andersen model, the predisposing factors that determined the use of both PC and $\mathrm{HC}$ services were age, in line with the existing literature [38-40, 42, 44, 46], and Spanish origin, which may be because people of other nationalities may have less access to health care and the enactment of Royal Decree Law 16/2012 in 2012, where it is described that foreign people in an irregular situation lost the right of access to standardized health care. The need factors that influenced the use of services at both levels of care were the need for palliative care, high risk, the number of chronic diseases and neoplasms.

For PC users, predisposing factors such as female gender were specifically associated with greater use of services, as is also reflected in the literature [36, 37]; to a greater extent, need factors such as being immobilized and presenting diseases that require polymedication and frequent monitoring or palliative care (diabetes mellitus, heart failure, stroke, dementia) influenced PC use, consistent with other studies $[38,39,46]$.

Among $\mathrm{HC}$ users, there was a lower use among immobilized patients and patients with dementia, which is explained because care for this group primarily entails home visits from PC services. In contrast, among $\mathrm{HC}$ users, greater use by patients with other diseases, such as depression, stood out, which coincides with the literature and is explained by the coexistence of these diseases with a variety of other chronic diseases that require hospital care, such as heart diseases, cerebrovascular accidents, cancer or diabetes [47] and COPD, which is one of the main reasons for visits to outpatient clinics and emergency rooms and hospital admissions [48, 49].

The risk levels are intended to guide $\mathrm{PC}$ and $\mathrm{HC}$ health professionals through assigning a specific intervention and coordinated level of care to each chronic patient, along with their knowledge of that patient and its context. This offers a more individualized and patient-centered attention based on the Kaiser Permanente stratification model. The goal for low risk patients is to slow the progression of the disease and prevent the patient from reaching higher levels of risk. To this end, the self-management of the disease and the education of a preventive nature and healthy habits are supported to avoid healthcare utilization. The goal for patients at medium risk is to slow progression by planning and managing the disease that combines self-management and professional care. The high risk patients have an increased utilization of healthcare services and the objective at this level is to reduce flare-ups and hospital admissions through comprehensive case management, with mainly professional care [24]. This ideal service selection correlates with current service utilization observed in the study with an increased healthcare services utilization in the high-risk level both in PC and $\mathrm{HC}$.

\section{Strengths and limitations}

As a cross-sectional study, the nature of the associations cannot be interpreted in terms of cause-effect. On the other hand, the existence of information biases due to mistakes or errors in diagnostic coding could limit the ability of the EMR to reflect real morbidity, but most ICPC-2- diagnostic codes and the rest of clinical and contacts information are recorded by the health professional responsible for the patient care. Additionally, the use of secondary clinical-administrative sources makes it possible to work with almost all individuals and not with partial samples or volunteers, which minimizes possible selection and memory biases. The existence of patients who may not be represented in the total population of the center because they were not contacted or did not use PC services due to having double insurance is unlikely to significantly alter the results since the proportion of people with a health care card in Madrid reaches 95\% [17]. In our study, there were no factors related to the service provider or organization that were considered likely to influence the use of the service, since those data are not available. However, most use of services is usually attributed to factors related to the user [49]. Regarding AMGs, doubts have been raised about their transparency, although this is a situation common to commercial classification tools, as stated by Huntley et al. [2]. As a result of this, it's not exactly known how specifically each different factor is weighted in the complexity index per CBM. Besides, AMGs have a clinicalcare management purpose that considers the complexity and morbidity of the patient but does not take into account other factors, such as their psychosocial and socioeconomic situation. Despite this, there has been strong agreement among classifiers, and AMGs have shown good predictive capacity [14]. The developers of AMGs have shown that they are a useful tool that allows the detection of comparable health centers and facilitates the study of variability in the consumption of resources and other clinical-organizational aspects [8]. Therefore, the Ministry of Health, Social Services and Equality is integrating them into the EMR of the National Health System in autonomous communities for the management of chronic patients $[8,20]$.

\section{Conclusions}

The characteristics and use of PC and $\mathrm{HC}$ services by chronic patients differed and varied according to the 
patients risk level, as determined by their AMG. PC service use was higher than $\mathrm{HC}$ use, although the use of both levels of care was high. The most frequent type of contact in PC was with a health care provider, specifically a family doctor, while in $\mathrm{HC}$, it was outpatient consultation and emergency care. The use corresponded with predisposing factors such as age and country of origin and, above all, with need factors such as immobility, high risk, the number and type of chronic diseases and the need for monitoring and palliative care.

\section{Supplementary Information}

The online version contains supplementary material available at https://doi. org/10.1186/s12913-021-07020-z.

\section{Additional file 1 .}

\section{Acknowledgements}

To the professionals of the Healthcare Centre Ciudad Jardín and the Research Unit of the Primary Care Management of Madrid for their methodological support.

\begin{abstract}
Authors' contributions
JBC: Conceptualization; Data curation; Formal analysis; Funding acquisition; Investigation; Methodology; Project administration; Resources; Software; Supervision; Validation; Visualization; Roles/Writing - original draft; Writing review \& editing. MSRO: Conceptualization; Data curation; Formal analysis; Investigation; Methodology; Validation; Visualization; Roles/Writing - original draft; Writing - review \& editing. MMC: Conceptualization; Investigation; Methodology; Validation; Visualization; Roles/Writing - original draft; Writing review \& editing. ACR: Conceptualization; Data curation; Formal analysis; Investigation; Methodology; Validation; Visualization; Roles/Writing - original draft; Writing - review \& editing. MBDO: Conceptualization; Data curation; Formal analysis; Investigation; Methodology; Validation; Visualization; Roles/ Writing - original draft; Writing - review \& editing. MTBM: Conceptualization; Data curation; Formal analysis; Investigation; Methodology; Validation; Visualization; Roles/Writing - original draft; Writing - review \& editing. MCLR: Conceptualization; Methodology; Validation; Visualization; Roles/Writing original draft; Writing - review \& editing. MAJS: Conceptualization; Methodology; Validation; Visualization; Roles/Writing - original draft; Writing review \& editing. The author(s) read and approved the final manuscript.
\end{abstract}

\section{Funding}

This project received a grant for the translation and publication of this paper from the Foundation for Biosanitary Research and Innovation in Primary Care (FIIBAP).

\section{Availability of data and materials}

The datasets generated and analysed during the current study are not publicly available due the belong to the Madrid Health Service electronic medical record but are available from the corresponding author on reasonable request.

\section{Declarations}

\section{Ethics approval and consent to participate}

The study has the approval of the Drug Research Ethics Committee of the La Princesa University Hospital and a favorable report from the Local Research Commission of the Primary Care Management of the Community of Madrid. All methods were performed in accordance with the relevant guidelines and regulations. The Drug Research Ethics Committee of the La Princesa University Hospital waived the need for consent because the manuscript does not contain any individual personal data since the data were obtained from a secondary database with anonymized and dissociated information as stipulated by current legislation at the time of the study.
Consent for publication

Not applicable.

\section{Competing interests}

The authors declare that they have no competing interests.

\section{Author details}

${ }^{1}$ Primary Care Investigation Unit, Gerencia Asistencial de Atención Primaria, Madrid, Spain. ${ }^{2}$ Foundation for Biosanitary Research and Innovation in Primary Care, Madrid, Spain. ${ }^{3}$ Faculty of Health. Universidad Camilo José Cela, Madrid, Spain. ${ }^{4}$ Healthcare Centre Ciudad Jardín, Gerencia Asistencial de Atención Primaria, Madrid, Spain. ${ }^{5}$ Healthcare Centre Fuencarral, Gerencia Asistencial de Atención Primaria, Madrid, Spain. ${ }^{6}$ Preventive Medicine Department, Hospital Virgen de la Salud. Complejo Hospitalario de Toledo, Toledo, Spain.

Received: 16 June 2021 Accepted: 13 September 2021

Published online: 03 October 2021

\section{References}

1. Ollero Baturone M, Orozco Beltrán D, Domingo Rico C, Román Sánchez P, López Soto A, Melguizo Jiménez M, et al. «Declaración de Sevilla» conferencia nacional para la atencion al paciente con enfermedades crónicas. Rev Clínica Española. 2011;211(11):604-6. https://doi.org/10.1016/j. rce.2011.09.007.

2. Huntley AL, Johnson R, Purdy S, Valderas JM, Salisbury C. Measures of multimorbidity and morbidity burden for use in primary care and community settings: a systematic review and guide. Ann Fam Med. 2012; 10(2):134-41. https://doi.org/10.1370/afm.1363.

3. Bengoa R. Empantanados. Rev Innov Sanit y Atención Integr. 2008;1:1-7.

4. Wolff $J$, Starfield B, Anderson G. Prevalence, expenditures, and complications of multiple chronic conditions in the elderly. Arch Intern Med. 2002;162(20):2269-76. https://doi.org/10.1001/archinte.162.20.2269.

5. Ministerio de Sanidad, Servicios Sociales e Igualdad. Sistema Nacional de Salud. España 2012 [monografía en Internet]. Madrid; 2012. Disponible en: https://www.mscbs.gob.es/en/organizacion/sns/docs/sns2012/SNS012 Espanol.pdf.

6. Terraza Núñez R, Vargas Lorenzo I, Vázquez Navarrete ML. La coordinación entre niveles asistenciales: una sistematización de sus instrumentos y medidas. Gac Sanit. 2006;20(6):485-95. https://doi.org/10.1157/13096516.

7. Fernández Moyano A, García Garmendia JL, Palmero Palmero C, García Vargas-Machuca B, Páez Pinto JM, Álvarez Alcina M, et al. Continuidad asistencial. Evaluación de un programa de colaboración entre Atención Hospitalaria y Atención Primaria. Rev Clínica Española. 2007;207:510-20.

8. Monterde D, Vela E, Clèries M. Los grupos de morbilidad ajustados: nuevo agrupador de morbilidad poblacional de utilidad en el ámbito de la atención primaria. Atención Primaria. 2016;48(10):674-82. https://doi.org/1 0.1016/j.aprim.2016.06.003.

9. González González Al, Miquel Gómez AM, Rodríguez Morales D, Hernández Pascual M, Sánchez Perruca L, Mediavilla HI. Concordancia y utilidad de un sistema de estratificación para la toma de decisiones clínicas. Atención Primaria. 2017:49(4):240-7. https://doi.org/10.1016/j.aprim.2016.04.009.

10. Humphries, R. Social care funding and the NHS - An impending crisis?, London: The King's Fund. International Federation of Social Work; 2012.

11. Grupo de trabajo Ministerio de Sanidad Servicios Sociales e Igualdad. Informe del proyecto de estratificación de la población por grupos de morbilidad ajustados (GMA) en el Sistema Nacional de Salud (2014-2016). 2018.

12. Orueta JF, Nuño-Solinis R, Mateos M, Vergara I, Grandes G, Esnaola S. Predictive risk modelling in the Spanish population: a cross-sectional study. BMC Health Serv Res. 2013;13(1):269. https:/doi.org/10.1186/1472-6963-13-269.

13. Hughes JS, Averill RF, Eisenhandler J, Goldfield NI, Muldoon J, Neff JM, et al. Clinical risk groups (CRGs). Med Care. 2004;42(1):81-90. https://doi.org/10.1 097/01.mlr.0000102367.93252.70.

14. Estupiñán-Ramírez M, Tristancho-Ajamil R, Company-Sancho MC, SánchezJanáriz H. Comparación de modelos predictivos para la selección de pacientes de alta complejidad. Gac Sanit. 2019;33(1):60-5. https://doi.org/1 0.1016/j.gaceta.2017.06.003

15. Arias-López C, Rodrigo Val MP, Casaña Fernández L, Salvador Sánchez L, Dorado Díaz A, Estupiñán RM. Validación del poder predictivo de los Grupos de Morbilidad Ajustada (GMA) respecto de otras herramientas de estratificación de la población; 2020. p. 94.3 de julio e202007079 
16. Monterde D, Vela E, Clèries M, García Eroles L, Pérez SP. Validity of adjusted morbidity groups with respect to clinical risk groups in the field of primary care. Aten Primaria. 2019;51(3):153-61. https://doi.org/10.1016/j.aprim.2017. 09.012.

17. Esteban-Vasallo M, Dominguez-Berjon M, Astray-Mochales J, Genova-Maleras R, Perez-Sania A, Sanchez-Perruca $L$, et al. Epidemiological usefulness of population-based electronic clinical records in primary care: estimation of the prevalence of chronic diseases. Fam Pract. 2009;26(6):445-54. https://doi. org/10.1093/fampra/cmp062.

18. Andersen RM. National Health Surveys and the behavioral model of health services use. Med Care. 2008;46(7):647-53. https://doi.org/10.1097/MLR. Ob013e31817a835d.

19. Ruiz-Rodríguez M, Valdez-Santiago R. Revisión sobre la Utilización de Servicios de Salud, 1996-2006. Rev Salud Pública. 2008;10(2):332-42. https:// doi.org/10.1590/S0124-00642008000200014.

20. Monterde D, Vela E, Clèries M, García Eroles L, Pérez SP. Validez de los grupos de morbilidad ajustados respecto a los clinical risk groups en el ámbito de la atención primaria. Atención Primaria. 2019;51(3):153-61. https://doi.org/10.1016/j.aprim.2017.09.012.

21. Barrio-Cortes J, del Cura-González I, Martínez-Martín M, López-Rodríguez C, Jaime-Sisó MÁ, Suárez-Fernández C. Grupos de morbilidad ajustados: características y comorbilidades de los pacientes crónicos según nivel de riesgo en Atención Primaria. Atención Primaria. 2020;52(2):86-95. https://doi. org/10.1016/j.aprim.2018.12.007.

22. Álvarez-del Arco D, Vicente Sánchez M, Alejos B, Pascual C, Regidor E. Construcción de un índice de privación para los barrios de Madrid y Barcelona. Rev Esp Salud Publica. 2013;87(4):317-29. https://doi.org/10.4321/ S1135-57272013000400003.

23. Consejería de Sanidad de la Comunidad de Madrid. Privación socioeconómica: índice compuesto de privación por secciones censales. Madrid: Dirección General de Atención Primaria; 2012.

24. Servicio Madrileño de Salud. Estrategia de Atención a Pacientes con Enfermedades Crónicas en la Comunidad de Madrid. Madrid: Consejería de Sanidad; 2013.

25. Monterde D, Vela E, Clèries M, Garcia-Eroles L, Roca J, Pérez-Sust P. Multimorbidity as a predictor of health service utilization in primary care: a registry-based study of the Catalan population. BMC Fam Pract. 2020;21(1): 39. https://doi.org/10.1186/s12875-020-01104-1.

26. Servicio Madrileño de Salud. Cartera de servicios estandarizados de Atención Primaria de Madrid. Madrid: Gerencia asistencial de Atención Primaria; 2014.

27. Rizza A, Kaplan V, Senn O, Rosemann T, Bhend H, Tandjung R. Age- and gender-related prevalence of multimorbidity in primary care: the swiss fire project. BMC Fam Pract. 2012;13(1):113. https://doi.org/10.1186/1471-22 96-13-113.

28. Rocca WA, Boyd CM, Grossardt BR, Bobo WV, Finney Rutten LJ, Roger VL, et al. Prevalence of multimorbidity in a geographically defined American population. Mayo Clin Proc. 2014;89(10):1336-49. https://doi.org/10.1016/j. mayocp.2014.07.010.

29. Marengoni A, Angleman S, Melis R, Mangialasche F, Karp A, Garmen A, et al. Aging with multimorbidity: a systematic review of the literature. Ageing Res Rev. 2011;10(4):430-9. https://doi.org/10.1016/j.arr.2011.03.003.

30. Salisbury C, Johnson L, Purdy S, Valderas JM, Montgomery AA Epidemiology and impact of multimorbidity in primary care: a retrospective cohort study. Br J Gen Pract. 2011;61(582):e12-21. https://doi.org/10.3399/ bjgp11X548929.

31. Glynn LG, Valderas JM, Healy P, Burke E, Newell J, Gillespie P, et al. The prevalence of multimorbidity in primary care and its effect on health care utilization and cost. Fam Pract. 2011;28(5):516-23. https://doi.org/10.1093/fa $\mathrm{mpra} / \mathrm{cmr} 013$.

32. Vedsted P, Olesen F. Social environment and frequent attendance in Danish general practice. Br J Gen Pract. 2005;55(516):510-5.

33. Martín-Fernández J, Gómez-Gascón T, del Cura-González MI, Tomás-García N, Vargas-Machuca C, Rodríguez-Martínez G. La calidad de vida relacionada con la salud como factor explicativo de la utilización de la consulta de medicina de familia: un estudio bajo el modelo conductual. Rev Esp Salud Publica. 2010;84(3):309-19. https://doi.org/10.1590/\$1135-572720100003 00007.

34. Ángel Bellón Saameño J, Delgado Sánchez A, de Dios Luna del Castillo J, Lardelli Claret P. Influencia de la edad y sexo sobre los distintos, tipos de utilización en atención primaria. Gac Sanit. 1995;9:343-53.
35. Martín-Fernández J, Rodríguez-Martínez G, Ariza-Cardiel G, Vergel Gutierrez MÁ, Hidalgo Escudero AV, Conde-López JF. Variables que condicionan la utilización de la consulta de enfermería en centros de salud de la Comunidad de Madrid. Rev Esp Salud Publica. 2013;87(4):383-92. https://doi. org/10.4321/S1135-57272013000400008.

36. Ortega Tallón MA, Roca FG, Iglesias RM, Jurado SJ. Pacientes hiperfrecuentadores de un centro de atención primaria: características sociodemográficas, clínicas y de utilización de los servicios sanitarios. Atención Primaria. 2004;33(2):78-85. https://doi.org/10.1016/S0212-6567(04 )79355-5.

37. Ministerio de Sanidad, Servicios Sociales e Igualdad. Actividad y Calidad de los Servicios Sanitarios, informe anual del SNS 2017. Madrid: Informes, Estudios e Investigación; 2019.

38. Carrera-Lasfuentes P, Abad JM, Aguilar-Palacio I, Rabanaque MJ. Comorbilidad como predictor de utilización de servicios sanitarios y mortalidad en pacientes con diabetes. Gac Sanit. 2015;29(1):10-4. https:// doi.org/10.1016/j.gaceta.2014.07.008.

39. Smith SM, Soubhi H, Fortin M, Hudon C, O'Dowd T. Managing patients with multimorbidity: systematic review of interventions in primary care and community settings. BMJ. 2012;345(sep03 1):e5205.

40. Sáez M. Condicionantes en la utilización de los servicios de atención primaria. Evidencias empíricas e inconsistencias metodológicas. Gac Sanit. 2003;17(5):412-9. https://doi.org/10.1016/S0213-9111(03)71778-6.

41. Van Minh H, Nawi N, Juvekar S, Razzaque A, Ashraf A, Hadi A, et al. Selfreported prevalence of chronic diseases and their relation to selected sociodemographic variables: a study in INDEPTH Asian sites, 2005. Prev Chronic Dis. 2008;5:A86.

42. Hernandez C, Jansa M, Vidal M, Nuñez M, Bertran MJ, Garcia-Aymerich J, et al. The burden of chronic disorders on hospital admissions prompts the need for new modalities of care: a cross-sectional analysis in a tertiary hospital. QJM An Int J Med. 2009;102(3):193-202. https://doi.org/10.1093/ qjmed/hen172.

43. Garin N, Olaya B, Perales J, Moneta MV, Miret M, Ayuso-Mateos JL, et al. Multimorbidity patterns in a national representative sample of the Spanish adult population. PLoS One. 2014;9(1):e84794. https://doi.org/10.1371/journa I.pone.0084794.

44. Coffey A, Leahy-Warren P, Savage E, Hegarty J, Cornally N, Day MR, et al. Interventions to Promote Early Discharge and Avoid Inappropriate Hospital (Re)Admission: A Systematic Review. Int J Environ Res Public Health. 2019; 16:2457.

45. Ministerio De Sanidad y Consumo. Informe Salud y Género 2005. Madrid; 2009.

46. Street M, Berry D, Considine J. Frequent use of emergency departments by older people: a comparative cohort study of characteristics and outcomes. Int J Qual Heal Care. 2018;30(8):624-9. https://doi.org/10.1093/intahc/ mzy062.

47. Lozano MJG, Berrocal DG-C, de Maya Matallana MC, Mendoza GC. E profesional y la organización en el uso de recursos de atención primaria. Atención Primaria. 2000;26(8):526-32. https://doi.org/10.1016/S02126567(00)78717-8.

48. Horn EK, Van Benthem TB, Hakkaart-Van Roijen L, Van Marwijk HWJ, Beekman ATF, Rutten FF, et al. Cost-effectiveness of collaborative care for chronically ill patients with comorbid depressive disorder in the general hospital setting, a randomised controlled trial. BMC Health Serv Res. 2007; 7(1):1-14. https://doi.org/10.1186/1472-6963-7-28.

49. Vanasse A, Courteau J, Courteau M, Benigeri M, Chiu YM, Dufour I, et al. Healthcare utilization after a first hospitalization for COPD: a new approach of state sequence analysis based on the " $6 \mathrm{~W}$ " multidimensional model of care trajectories. BMC Health Serv Res. 2020;20(1):1-15. https://doi.org/10.11 86/s12913-020-5030-0.

\section{Publisher's Note}

Springer Nature remains neutral with regard to jurisdictional claims in published maps and institutional affiliations. 